\title{
The Roles of the Caudate Nucleus in Human Classification Learning
}

\author{
Carol A. Seger and Corinna M. Cincotta \\ Department of Psychology, Colorado State University, Fort Collins, Colorado 80523
}

The caudate nucleus is commonly active when learning relationships between stimuli and responses or categories. Previous research has not differentiated between the contributions to learning in the caudate and its contributions to executive functions such as feedback processing. We used event-related functional magnetic resonance imaging while participants learned to categorize visual stimuli as predicting "rain" or "sun." In each trial, participants viewed a stimulus, indicated their prediction via a button press, and then received feedback. Conditions were defined on the bases of stimulus-outcome contingency (deterministic, probabilistic, and random) and feedback (negative and positive). A region of interest analysis was used to examine activity in the head of the caudate, body/tail of the caudate, and putamen. Activity associated with successful learning was localized in the body and tail of the caudate and putamen; this activity increased as the stimulus- outcome contingencies were learned. In contrast, activity in the head of the caudate and ventral striatum was associated most strongly with processing feedback and decreased across trials. The left superior frontal gyrus was more active for deterministic than probabilistic stimuli; conversely, extrastriate visual areas were more active for probabilistic than deterministic stimuli. Overall, hippocampal activity was associated with receiving positive feedback but not with correct classification. Successful learning correlated positively with activity in the body and tail of the caudate nucleus and negatively with activity in the hippocampus.

Key words: caudate; hippocampus; learning; visuomotor associations; categorization; fMRI

\section{Introduction}

Learning associations between stimuli and responses or categories is an important ability across species (Wise and Murray, 2000), and the striatum, particularly the caudate nucleus, plays a key role in such learning. Functional magnetic resonance imaging (fMRI) and positron emission tomography studies in humans have found caudate activity in many tasks ranging in complexity from learning to respond to individual stimuli with arbitrary responses (Toni et al., 2002) to classifying multiple stimuli with varying features into categories (Poldrack et al., 1999, 2001; Seger and Cincotta, 2002; Volz et al., 2003). Performance on these tasks is impaired in patients with compromised striatal functioning (Packard and Knowlton, 2002). Common features of task that involve the caudate include the presence of feedback and indication of decisions via distinct motor responses. Tasks with these characteristics are learned by a system that is behaviorally and neurally dissociable from two other human category learning systems (Ashby and Casale, 2003): categorizing on the basis of verbalizable rules, reliant on lateral frontal systems (Smith et al., 1998; Seger et al., 2000; Filoteo et al., 2005b), and learning single categories consisting of distortions of a prototypical stimulus, reliant on extrastriate visual areas (Reber et al., 1998a,b, 2003; Aizenstein et al., 2000)

\footnotetext{
Received March 14, 2004; revised Jan. 18, 2005; accepted Jan. 18, 2005.

This research was supported by National Institute of Mental Health Grant 63784 .

Correspondence should be addressed to Carol A. Seger, Department of Psychology, Colorado State University, Fort Collins, C0 80523. E-mail: seger@lamar.colostate.edu.

D01:10.1523/JNEUROSCI.3401-04.2005

Copyright $\odot 2005$ Society for Neuroscience $\quad$ 0270-6474/05/252941-11\$15.00/0
}

A puzzling discrepancy is that the main area of activity reported in human functional imaging studies is typically in the head of the caudate (Table 1), whereas research in monkeys shows the body and tail of the caudate to be crucial (Brown et al., 1995; Teng et al., 2000; Fernandez-Ruiz et al., 2001). Although there is a large degree of divergence in projections from the cortex to the striatum (Mink, 1996; Wise et al., 1996), it is generally accepted that the head and tail of the caudate participate in functionally dissociable corticostriatal loops. The head of the caudate interacts with dorsolateral prefrontal cortex as part of the "cognitive" corticostriatal loop, whereas the tail interacts with inferior temporal areas as part of the "visual" corticostriatal loop (Middleton and Strick, 1996; Lawrence et al., 1998). Many functional imaging studies link activity in the head of the caudate with executive functions related to receiving feedback, as summarized in Table 2. Activity in the ventral striatum is also associated with error in prediction (Berns et al., 2001). Feedback-processing demands are typically high in classification tasks, particularly when stimulus-response contingencies are probabilistic and perfect performance cannot be achieved.

Our primary goal was to use event-related fMRI to separate striatal activation linked to learning of associations from activation resulting from processing feedback. We also examined interactions between the caudate and the medial temporal lobe. Learning mediated by the caudate nucleus is dissociable from memory mediated by the medial temporal lobe. Patients with amnesia show relatively preserved learning on categorization tasks with feedback (Knowlton et al., 1994; Reber et al., 1996; Myers et al., 2003) (but see Hopkins et al., 2004). Both animal and human research finds an antagonistic relationship be- 
Table 1. Striatal activation foci in functional imaging studies of visual categorization and visuomotor learning

\begin{tabular}{|c|c|c|c|c|c|c|c|c|}
\hline Reference & Right $x, y, z$ & Left $x, y, z$ & Striatal region & $\begin{array}{l}\text { Total number } \\
\text { of different } \\
\text { stimuli used }\end{array}$ & $\begin{array}{l}\text { Total number of } \\
\text { categories or } \\
\text { different possible } \\
\text { responses }\end{array}$ & Feedback? & $\begin{array}{l}\text { Contingency } \\
\text { type }\end{array}$ & Task name \\
\hline Aron et al., 2004 & $\begin{array}{l}6,-12,-6 \\
9,12,0\end{array}$ & $\begin{array}{l}-6,-12,-6 \\
-9,9,0\end{array}$ & Head, ventral & 14 & 2 & Yes & $\mathrm{P}$ & Probabilistic classification \\
\hline Haruno et al., 2004 & $12,3,10$ & $0,-3,0$ & Head/body & 2 & 2 & Yes & $\mathrm{P}$ & Stochastic decision \\
\hline Lieberman et al., 2004 & $\begin{array}{l}12,-2,22 \\
14,16,0 \\
17,-4,24 \\
34,-34,2\end{array}$ & $-32,-10,20$ & $\begin{array}{l}\text { Head } \\
\text { Head } \\
\text { Body } \\
\text { Tail } \\
\text { Putamen }\end{array}$ & 32 & 2 & No & $\mathrm{P}$ & Artificial grammar \\
\hline 0'Doherty et al., 2003, 2004 & & $-8,22,0$ & Head & 2 & 2 & Yes & $P$ & Instrumental conditioning \\
\hline Poldrack et al., 1999 & $6,2,0$ & & Head & 14 & 2 & Yes & $P$ & Probabilistic classification \\
\hline Poldrack et al., 2001 & $9,6,21$ & $-12,3,21$ & Head/body & 14 & 2 & Yes & $\mathrm{P}$ & Probabilistic classification \\
\hline Seger and Cincotta, 2002 & $24,-2,-2$ & $-22,-8,0$ & Head, putamen & $U$ & 2 & Yes & $\mathrm{P}$ & Information integration \\
\hline Toni and Passingham, 1999 & $24,18,16$ & $-12,8,18$ & Head & 4 & 4 & Yes & D & Visuomotor associations \\
\hline Toni et al., 2001a & & $-18,18,4$ & Head & 4 & 4 & Yes & D & Visuomotor associations \\
\hline Toni et al., 2001b & & $-14,6,-2$ & Putamen & 4 & 4 & Yes & D & Visuomotor associations \\
\hline Vogels et al., 2002 & & $-24,18,0$ & Head & U & 3 & Yes & $P$ & Dot pattern classification \\
\hline
\end{tabular}

$U$ indicates that the potential size of the stimulus set was unlimited, and novel exemplars were presented on each trial. D, Deterministic; P, probabilistic.

Table 2. Activation in classification tasks associated with feedback or set shifting

\begin{tabular}{|c|c|c|c|c|c|}
\hline \multirow[b]{2}{*}{ Reference } & \multicolumn{3}{|l|}{ Striatal ROls } & \multirow{2}{*}{$\frac{\text { Feedback or shift comparison }}{\text { Shifting: objects }>\text { rule }}$} & \multirow[b]{2}{*}{ Task name } \\
\hline & Right $x, y, z$ & Left $x, y, z$ & Striatal region & & \\
\hline \multirow[t]{3}{*}{ Monchi et al., 2001} & $12,12,3$ & $-16,14,4$ & Head & Negative feedback $>$ control & WCST \\
\hline & $4,6,4$ & $-8,8,4$ & Head & Negative $>$ positive feedback & \\
\hline & & $-26,-10,4$ & Putamen & Matching after negative feedback & \\
\hline \multirow[t]{3}{*}{ Monchi et al., 2004} & & $-12,2,2$ & Head & Negative feedback $>$ control & WCST \\
\hline & & $-10,-6,2$ & Head & Negative $>$ positive feedback & Healthy elderly participants \\
\hline & $26,-4,4$ & $-24,-8,2$ & Putamen & Matching after negative feedback & \\
\hline \multirow[t]{2}{*}{ Rogers et al., 2000} & & $-6,16,8$ & Head & Reversal $>$ other shifts & $\begin{array}{l}\text { Three kinds of shifting tasks: intradimensional, } \\
\text { extradimensional, and reversal }\end{array}$ \\
\hline & & $-16,8,20$ & Head & & \\
\hline Cools et al., 2002 & & $-10,8,-4$ & Head & Final reversal error trial & Reversal learning \\
\hline Cools et al., 2004 & $9,-3,-3$ & & Head & Shifting $>$ no shift & Shifting between objects and/or rules \\
\hline
\end{tabular}

tween striatal and medial temporal lobe systems (Poldrack and Packard, 2003).

\section{Materials and Methods}

Participants. Participants were 15 members of the Stanford community (Stanford University, Stanford, CA) (seven males, eight females) with an average age of 24 years (range, 19-33). Participants were right handed, fluent speakers of English, met the criteria for MRI scanning (no metallic implants, no claustrophobia, head size compatible with the custom head coil), and were neurologically healthy (no known neurological or psychiatric injury or disease; not taking any psychoactive medication or drugs). Two of the participants completed only the first functional imaging scan because of limited scanning time. Functional data from the first scan from a third participant was lost because of technical problems.

Materials. In the behavioral task, participants viewed arbitrary visual patterns (Fig. 1) and used them to predict one of two possible outcomes, "rain" or "sun." Each stimulus was formed from five line segments of equal length; two lines were vertical, two were $\sim 105^{\circ}$ from vertical, and one was $45^{\circ}$ from vertical. The lines were arranged in arbitrary relation to each other to form visually distinct patterns. The stimuli were not designed to have any features in common across categories.

The relationship between each stimulus and the two response categories varied in probability. Two stimuli were assigned to the deterministic condition; each was consistently paired with the same outcome (one stimulus was always paired with sun, and the other stimulus was always paired with rain). Four stimuli were assigned to the probabilistic condition, in which each was paired with one outcome for 80 or $90 \%$ of the time and the opposite outcome for 10 or $20 \%$ of the time (one stimulus was $90 / 10 \mathrm{sun} / \mathrm{rain}$, one was $80 / 20 \mathrm{sun} / \mathrm{rain}$, one was $90 / 10 \mathrm{rain} / \mathrm{sun}$, and one was 80/20 rain/sun). Two stimuli were assigned to the random condition, in which they were paired with each outcome for $50 \%$ of the time. The stimuli were randomly assigned to each condition for each participant to avoid specific item effects. Additionally, there was a baseline condition, in which participants viewed a large black plus sign and made no response.

Procedure. A "weather prediction" cover story was used, in which participants were asked to pretend to be meteorologists and use the visual stimuli to predict rain or sun. In each trial, one stimulus appeared on the screen for $2000 \mathrm{~ms}$, during which the participants made a button press response. The stimulus was followed by a blank screen for $250 \mathrm{~ms}$, the feedback "Correct!" or "Incorrect" for $500 \mathrm{~ms}$, and finally a blank screen again for $250 \mathrm{~ms}$. During baseline trials, the baseline stimulus alone appeared for a variable amount of time (typically between 1500 and 4500 $\mathrm{ms})$. The presentation sequence for all trials and the variable stimulus presentation times for baseline trials were determined by using optseq2 version 2.0 (Dale, 1999) (available at http://surfer.nmr.mgh.harvard. edu/optseq) to provide an optimum-jittered sequence for event-related analysis. The resulting order was pseudorandom; stimuli of the same type occasionally appeared in adjacent positions but were not purposefully grouped by type. Participants were not informed that the stimulus-out- 

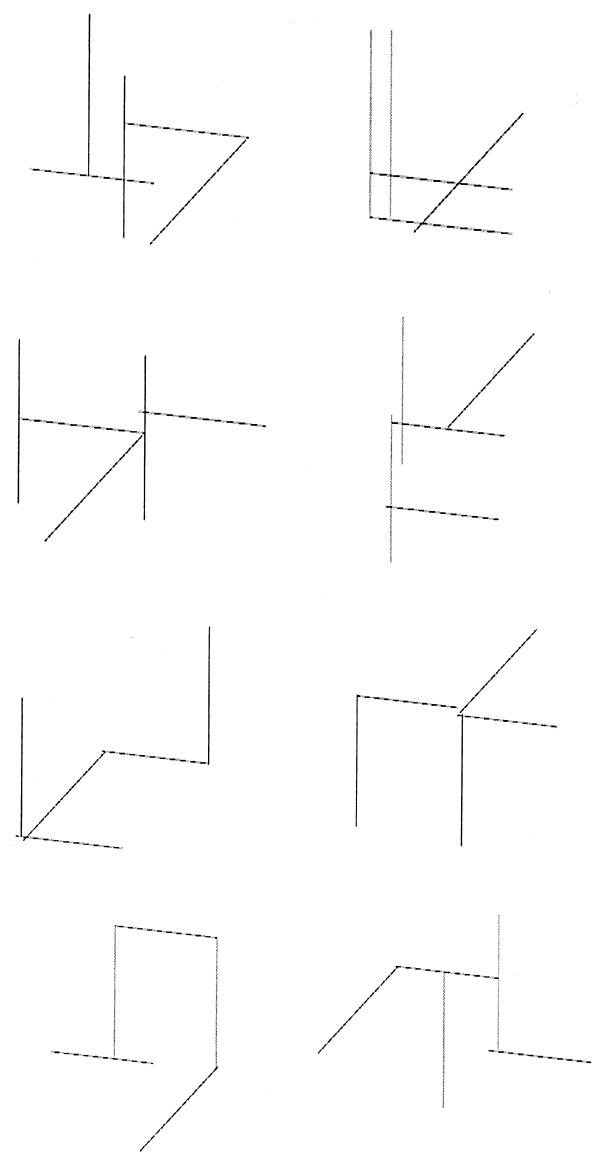

Figure 1. Stimuli used in the experiment.

come contingencies would differ across stimuli and were not explicitly informed of the trial type during each trial.

There were 640 trials: 160 baseline (Base), 120 deterministic (Det), 240 probabilistic (Prob), and 120 random (Ran). Because of scanner memory limitations, the task was broken into two 15 min scans with 326 trials in scan 1 and 314 trials in scan 2; the total number of trials differed across scans because of variations in stimulus timing dictated by optseq2. To examine changes in activity of areas across learning, we divided the study into three blocks with approximately equal numbers of each trial type in each block (e.g., 53 baseline trials, 40 deterministic trials, 80 probabilistic trials, and 40 random trials). This division provided the best tradeoff between maximizing trials per block for statistical power in the imaging contrasts and the desire to follow changes in learning.

fMRI image acquisition. Imaging was performed with a custom-built whole-head coil in a 3.0 tesla MRI Signa LX Horizon Echospeed (General Electric Medical Systems, Milwaukee, WI). Head movement was minimized for participants using a "bite-bar" formed with the participant's dental impression. In addition to the functional scans, three anatomical scans were performed: a coronal T1-weighted localizer scan, a threedimensional high-resolution T1-weighted spoiled gradient echo scan with 124 contiguous $1.5 \mathrm{~mm}$ slices [minimum full echo time (TE); $30^{\circ}$ flip angle; $24 \mathrm{~cm}$ field of view; $256 \times 256$ acquisition matrix] and a in-plane anatomical T1-weighted spin-echo scan with 22 contiguous 5 $\mathrm{mm}$ axial slices [minimum full TE; $500 \mathrm{~ms}$ repetition time (TR); $24 \mathrm{~cm}$ field of view; $256 \times 256$ acquisition matrix]. Functional scanning was performed using a $\mathrm{T} 2{ }^{\star}$-sensitive gradient echo spiral in-out pulse sequence (Glover and Law, 2001; Preston et al., 2004) (30 ms TE; $1500 \mathrm{~ms}$ TR; $65^{\circ}$ flip angle; $24 \mathrm{~cm}$ field of view; $64 \times 64$ acquisition matrix) of the same 22 contiguous $5 \mathrm{~mm}$ axial slices as the in-plane images.

Stimuli were presented using a magnet-compatible projector (Resonance Technology, Van Nuys, CA) that back-projects visual images onto a screen mounted above the participant's head. E-prime software (Psy- chology Software Tools, Pittsburgh, PA) running on a personal computer was used to generate visual stimuli and control experimental parameters. Responses were obtained using a fiber-optic finger-switch response system.

Image processing. Image analysis was performed using BrainVoyager 20004.9 , with the exception of the striatal region of interest (ROI) analyses, which were performed using BrainVoyager QX 1.0.9 (Brain Innovation, Maastricht, The Netherlands). The functional data were first subjected to preprocessing, consisting of three-dimensional motion correction, slice scan time correction, and temporal data smoothing with a high-pass filter of three cycles in the time course and linear trend removal. Each participant's high-resolution anatomical image was normalized to the Montreal Neurological Institute brain template. The normalization process in BrainVoyager consists of two steps: an initial rigid body translation into the anterior commissure-posterior commissure plane, followed by an elastic deformation into the standard space performed on 12 individual subvolumes. The resulting set of transformations was applied to the participant's functional image volumes to form volume time course representations to be used in subsequent statistical analyses. Finally, the volume time course representations were spatially smoothed with a Gaussian kernel, full-width at half-maximum of $6.0 \mathrm{~mm}$.

Statistical analysis. Trials were first divided by stimulus types: Base, Det, Prob, and Ran. The categorization trial types were further divided into eight subcategories based on the correctness of the response and the feedback given to participants. Deterministic trials were divided into those that the participants classified correctly (in accordance with the dominant category assignment) and received positive feedback (Det-CP) and those that the participants classified incorrectly and received negative feedback (Det-IN). Probabilistic stimuli were classified both in terms of whether the participant responded correctly (in accordance with the dominant category assignment) and in terms of the feedback received by the participants. In Prob-CP trials, stimuli were classified correctly and the participant received positive feedback. In Prob-IN trials, the stimuli were classified incorrectly and the participant received negative feedback. In Prob-CN trials, participants classified the stimulus correctly but received negative feedback. Finally, in Prob-IP trials, the stimulus was classified incorrectly but the subject received positive feedback. For random stimuli, it was not possible to be correct or incorrect in the sense of responding according to the dominant assignment. Therefore, random stimuli were divided into two categories: Ran-P (positive feedback) and Ran-N (negative feedback). There were very few trials in three of the conditions (Det-IN, Prob-CN, and Prob-IP), and thus these conditions were not subjected to statistical analysis.

Because of the a priori prediction that striatal structures would be important for categorization, the primary statistical analysis was limited to eight ROIs in the striatum: the right and left head of the caudate nucleus, the right and left body/tail of the caudate nucleus, the right and left putamen, and the right and left ventral striatum. These areas were defined anatomically using a single subject's high-resolution normalized anatomical image and were confirmed to encompass the structure in all subjects. The ROIs were drawn with generous margins to ensure coverage of each structure across subjects; thus, the borders of the ROIs extended into surrounding white matter and ventricles but not into gray matter areas such as the thalamus, insula, or globus pallidus. The resulting ROIs are shown in Figure 2. The head of the caudate and putamen ROIs were limited to parts of these nuclei considered to be in the dorsal striatum. The ventral striatum is defined in primates to include the rostral ventral caudate and putamen nuclei in addition to the nucleus accumbens (Haber et al., 2000). In humans, the dividing line between dorsal and ventral is approximately $z=-1$. Studies that have found ventral striatum activity have typically localized it to $z=-4$ or -8 (Berns et al., 2001; McClure et al., 2004; O’Doherty et al., 2004).

The ROI general linear model tool of BrainVoyager QX 1.0.9 was used to analyze contrasts between conditions, separately within each ROI. This tool calculates the average time course of all voxels in the ROI for each subject, compares the time course across subjects and conditions in an ANOVA with subjects treated as random effects, and computes $t$ and $p$ values for contrasts of interest between specified conditions. Only contrasts with potential theoretical interest were analyzed. 


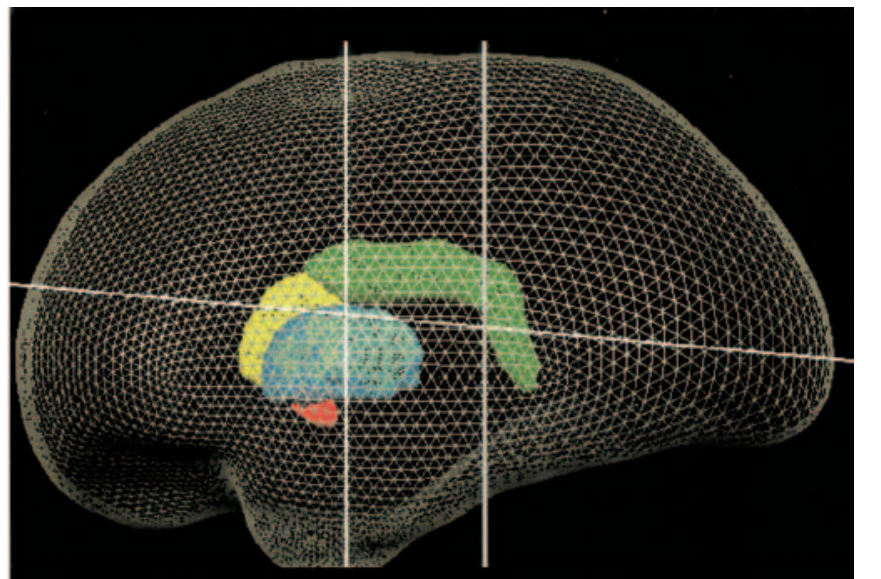

In addition to the ROI analysis of striatal structures, an exploratory whole-brain analysis was performed to identify additional structures that may be associated with classification learning. BrainVoyager was used to analyze contrasts between conditions. First, a model of the hemodynamic response for each condition was formed by convolving a prototypical hemodynamic response function with the time course of the conditions. Then, conditions were compared using the general linear model with separate subject predictors and subjects treated as random effects. Again, only contrasts with potential theoretical interest were analyzed. Given the exploratory nature of this analysis, the threshold was set at $p<$ 0.005 , uncorrected, which corresponded to a $t$ value threshold of 3.3 . This threshold was chosen to minimize "misses" of potentially interesting cortical areas that would not be activated using a higher threshold. However, this choice of threshold also increases the risk of "false positives" and should be kept in mind when interpreting the results.

Changes in brain activity across blocks were examined within certain ROIs. As detailed in Table 3, one of the conditions examined in the striatal ROI and whole-brain analysis, Prob-IN, had few or no trials per block for many participants. Thus, time course analyses were limited to the following conditions: Base, Det-CP, Prob-CP, Ran-P, and Ran-N For the striatum, ROIs were defined anatomically, as described above. For areas identified in the exploratory whole-brain analysis (left superior frontal gyrus and right hippocampus), ROIs were defined based on functionally activated clusters using the tools in BrainVoyager. The ROI is identified via a region-growing process, which starts with a central voxe indicated by a mouse click and spreads to suprathreshold adjacent voxels, stopping at the boundaries of the functional cluster. The percentage of signal change within each ROI was calculated for each participant, for each block of each condition, using the event-related averaging functions and the ROI analysis function. The average percentage of signal change measured from trial onset was calculated from $4-8 \mathrm{~s}$, inclusive; this time period was chosen to encompass the peak of the hemodynamic response function, which is typically $\sim 6 \mathrm{~s}$ after stimulus onset.

\section{Results}

\section{Behavioral results}

Classification accuracy across blocks for probabilistic, deterministic, and random trials is plotted in Figure 3. For probabilistic stimuli, being correct was defined in terms of whether the participants responded in accordance with the dominant assignment of the stimulus, rather than in terms of the feedback received, because this measure best reflects learning of the contingencies. A $3 \times 3$ block by stimulus type (deterministic, probabilistic, random) ANOVA with the dominant assignment accuracy values as the dependent measure revealed a main effect of block $\left(F_{(2,24)}=\right.$ $11.2 ; p<0.0005)$, a main effect of stimulus type $\left(F_{(2,24)}=40.0\right.$; $p<0.0001)$, and an interaction between block and stimulus type $\left(F_{(4,24)}=5.6 ; p<0.001\right)$. A visual inspection of the graphs in Figure 3 indicated that the interaction between block and stimu-

Figure 2. Three-dimensional rendering of the eight striatal regions of interest, viewed from the left, from above, and from the front. Green, Body and tail of the caudate; blue, head of the caudate; yellow, putamen; red, ventral striatum. White lines indicate the planes of $z=0, x=0, y=0$, and $y=-20$, as used in the brain atlas in the study by Talairach and Tournoux (1988). The putamen ROIs extended inferiorally to superiorally from $z=-1$ to $z=15$, anteriorally to posteriorally from $y=$ -15 to $y=+15$, and laterally from $x= \pm 15$ to \pm 33 . Within the caudate nucleus, the border between the head and the body/tail ROls was along an oblique plane angled at $45^{\circ}$ from horizontal running between the lines defined by $y=0, z=14$ and $y=10, z=24$. The head of the caudate ROIs extended inferiorally to $z=-1$ and laterally from the ventricles to $x= \pm 13$. The body/tail of the caudate ROls extended superiorally to $z=28$, inferiorally in the body portion to $z=16$, in the tail portion to $z=-3$, laterally from approximately $x=9$ to $x=22$ in the body region, and posteriorally to $y=-38$ at the tip of the tail. The ventral striatum ROI encompassed the nucleus accumbens and the mostinferior portions of the caudate and putamen; it extended in the inferior-superior dimension from $z=-2$ to $z=-10$

Table 3. Trials in each condition

\begin{tabular}{llcc}
\hline & Block 1 [mean (range)] & Block 2[mean (range)] & Block 3 [mean (range)] \\
\hline Det-CP & $24.9(13-36)$ & $27.8(13-37)$ & $38.3(18-44)$ \\
Det-IN $^{a}$ & $13.7(2-24)$ & $8.6(0-24)$ & $6.7(1-27)$ \\
Prob-CP & $51.8(39-66)$ & $50.5(31-64)$ & $55.2(44-63)$ \\
Prob-CN $^{a}$ & $8.6(6-12)$ & $10.3(8-13)$ & $10.3(8-11)$ \\
Prob-IP $^{a}$ & $3.4(0-9)$ & $3.1(0-10)$ & $0.8(0-3)$ \\
Prob-IN & $24.6(10-41)$ & $13.6(1-30)$ & $7.6(0-19)$ \\
Ran-N & $20.7(17-23)$ & $21(15-25)$ & $18.6(10-23)$ \\
Ran-P & $20.3(18-24)$ & $19.7(16-25)$ & $18.9(15-24)$
\end{tabular}

${ }^{a}$ Conditions not analyzed because of insufficient trials.

${ }^{b}$ Condition excluded from the time course analyses because of low numbers of trials in block 2 and block 3 . 


\section{Learning Across Blocks}
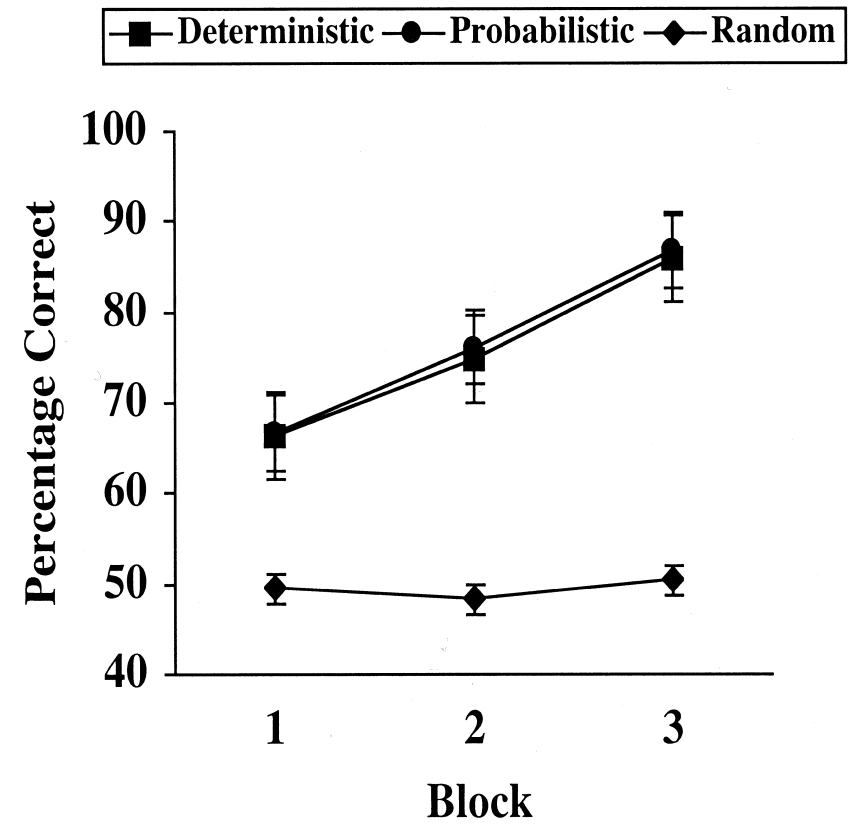

Figure 3. The percentage correct across blocks for deterministic, probabilistic, and random stimuli, with correct defined as classifying the stimulus consistent with its dominant assignment, is shown.

lus type was driven by the (expected) lack of learning of random stimulus-outcome associations. To examine differences in learning between deterministic and probabilistic stimuli, we performed a $3 \times 2$ block $\times$ stimulus type ANOVA, which indicated only a main effect of block $\left(F_{(2,12)}=13.4 ; p<0001\right)$, with no main effect of stimulus type or interaction of block by type, $F \mathrm{~s}<1.0$. We conclude that deterministic and probabilistic stimuli were learned equally well, and accuracy increased across blocks for both.

\section{Striatal ROI analyses}

Areas associated with classification: Det-CP and Prob-CP $>$ Base We first examined correct classification of both deterministic and probabilistic stimuli in comparison with the baseline task. Only correct trials were examined to avoid variability in the proportions of correct responses and negative feedback across stimulus types; however, each trial does include feedback. Contrasts were calculated separately for each striatal ROI, but all are shown in Figure 4 for convenience. Activation was significantly higher during classification than baseline in the right body and tail of the caudate $\left[t_{(14)}=5.61 ; p<0.0001\right]$, the left body and tail of the caudate $\left[t_{(14)}=3.48 ; p<0.005\right]$, the right putamen $\left[t_{(14)}=3.71\right.$; $p<0.005]$, and the left putamen $\left[t_{(14)}=3.42 ; p<0.0005\right]$. Activation did not differ between classification and baseline for the right or left head of the caudate.

As shown in Figure 5, activity in the right and left body and tail of the caudate and right and left putamen ROIs followed a very similar pattern across blocks. In block one, activity during classification was at or below baseline. In blocks two and three, activity during classification rose above baseline activity. As shown in Table 4, activity in the right body and tail significantly positively correlated with accuracy during the second half of the experiment; there was a trend toward a correlation between the left body/tail, right putamen, and left putamen and accuracy during

\section{Classification across striatal ROIs}

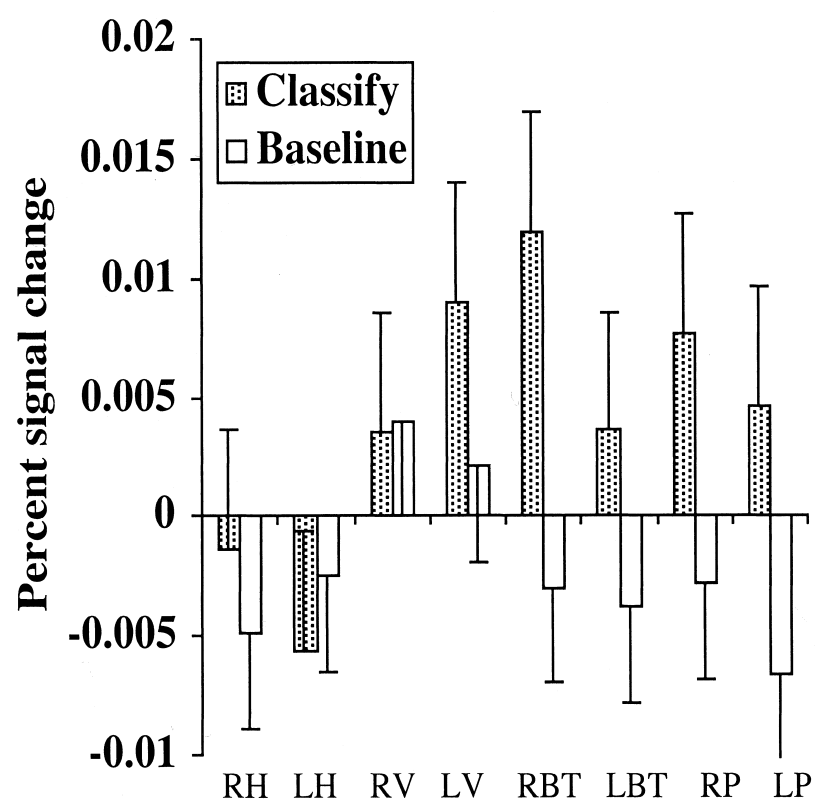

Figure 4. Percentage of signal change during classification (Det-CP and Prob-CP combined) and baseline trials in the eight striatal ROls: right head (RH) and left head (LH) of the caudate, right ventral striatum (RV) and left ventral striatum (LV), right body and tail (RBT) and left body and tail (LBT) of the caudate, and right putamen (RP) and left putamen (LP).
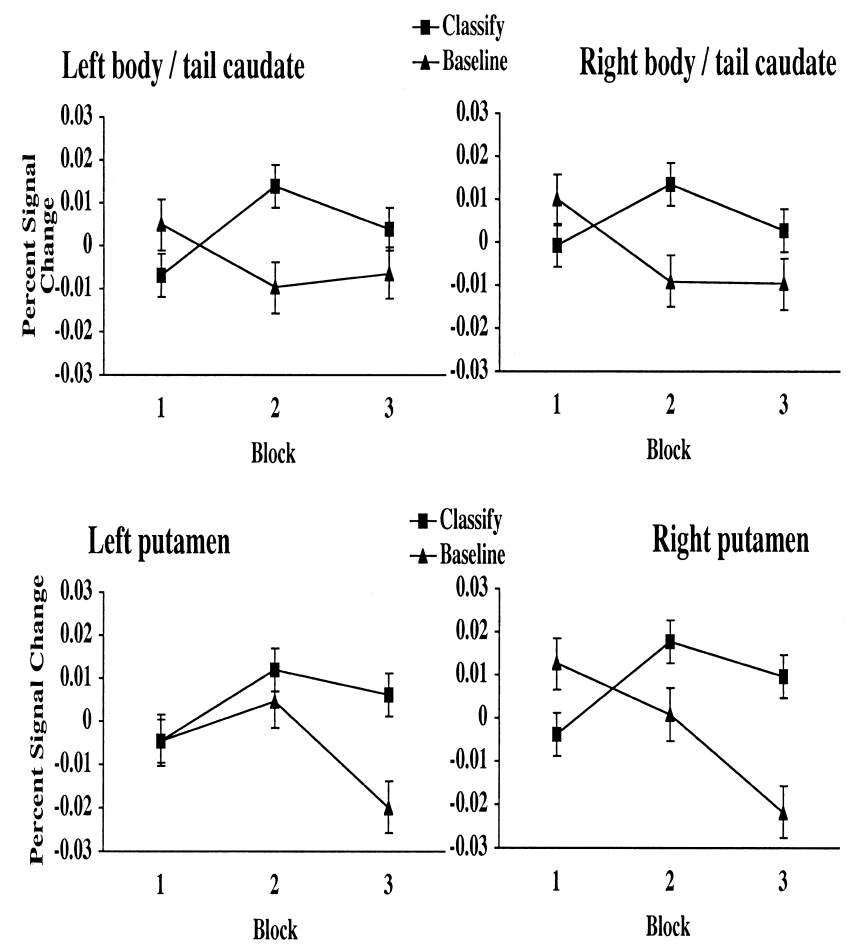

Figure 5. The percentage of signal change in the right and left body and tail of the caudate nucleus and right and left putamen across blocks for classification (Det-CP and Prob-CP combined) and baseline stimulus trials is shown.

the second half. Thus, participants who were more successful in learning to categorize stimuli recruited the body and tail of the caudate and putamen to a greater degree than participants who were less successful at learning. 
Table 4. Correlation between accuracy and regional blood flow in the caudate body and tail, putamen, and the hippocampus

\begin{tabular}{lcc}
\hline & Accuracy & \\
\cline { 2 - 3 } & First half & Second half \\
\hline Right body tail & 0.33 & $0.56^{*}$ \\
Left body tail & 0.19 & $0.47^{* *}$ \\
Right putamen & 0.15 & $0.47^{* *}$ \\
Left putamen & 0.16 & $0.49^{* *}$ \\
Right hippocampus & $-0.55^{*}$ & -0.24 \\
\hline
\end{tabular}

${ }^{*} p<0.05 ;{ }^{* *} p<0.1$. Accuracy was calculated in terms of consistency with the dominant category assignment for probabilistic stimuli. Responses to random stimuli were not included in the accuracy measure.

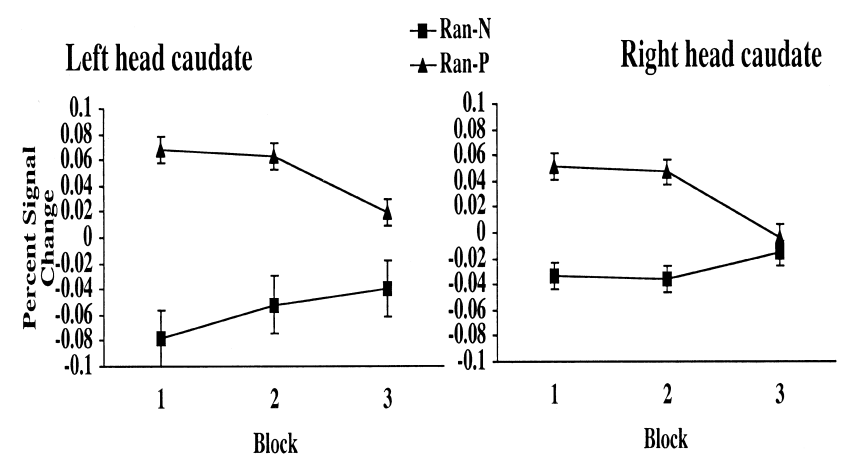

\section{Feedback activation across striatal ROIs}

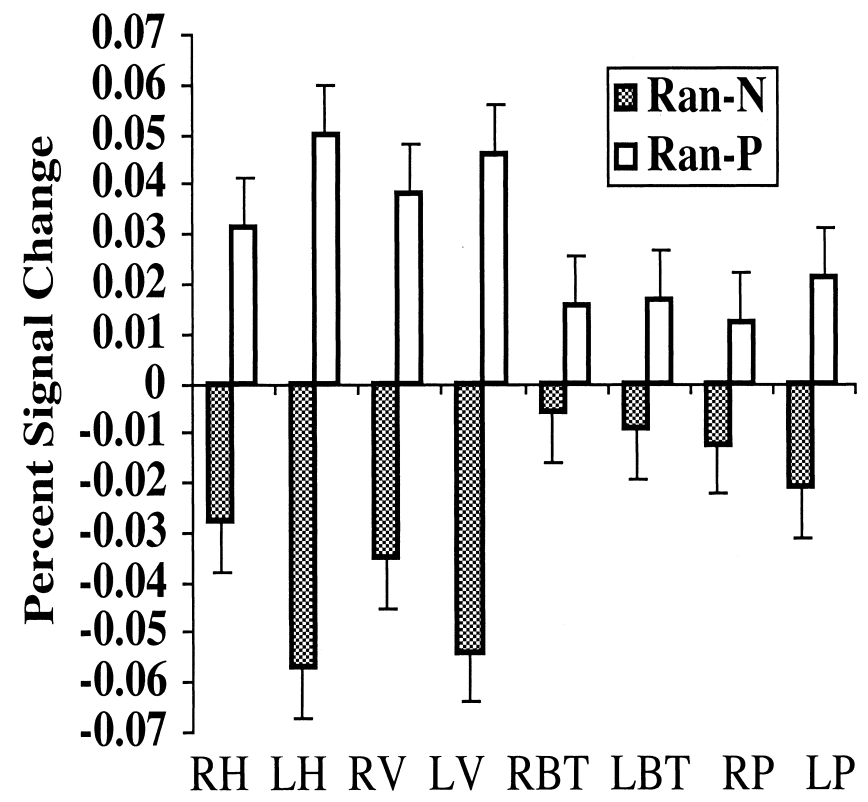

Figure 6. Percentage of signal change in the Ran-P and Ran-N conditions in the eight striatal ROls: right head $(\mathrm{RH})$ and left head $(\mathrm{LH})$ of the caudate, right ventral striatum (RV) and left ventral striatum (LV), right left body and tail (RBT) and left body and tail (LBT) of the caudate, and right putamen (RP) and left putamen (LP).

\section{Effect of stimulus contingency: pairwise comparisons between}

Det-CP, Prob-CP, and Ran-P

Activation was significantly higher for Prob-CP than for Det-CP in the left body and tail of the caudate $\left[t_{(14)}=2.36 ; p<0.05\right]$ and showed a trend toward higher activation in the right body and tail of the caudate $\left[t_{(14)}=1.84 ; p=0.086\right]$. Activation did not differ between Prob-CP and Det-CP in the right or left putamen or the right or left head of the caudate $(p>0.1)$. Activation did not significantly differ between Ran-P and either Prob-CP or Det-CP in any of the eight striatal ROIs $(p>0.1)$.

Effect of feedback: Ran-P $>$ Ran-N

There was greater activity for Ran-P than for Ran- $\mathrm{N}$ in all eight striatal ROIs: right head of the caudate $\left[t_{(14)}=3.49 ; p<0.005\right]$, left head of the caudate $\left[t_{(14)}=2.59 ; p<0.05\right]$, right body and tail of the caudate $\left[t_{(14)}=2.46 ; p<0.05\right]$, left body and tail of the caudate $\left[t_{(14)}=2.2 ; p<0.05\right]$, right putamen $\left[t_{(14)}=3.75 ; p<\right.$ $0.005]$, left putamen $\left[t_{(14)}=3.1 ; p<0.01\right]$, right ventral striatum $\left[t_{(14)}=3.39 ; p<0.005\right]$, and left ventral striatum $\left[t_{(14)}=3.75\right.$; $p<0.005]$. As shown in Figure 6, the difference between blood oxygen level-dependent signal in Ran-P and Ran-N trials was

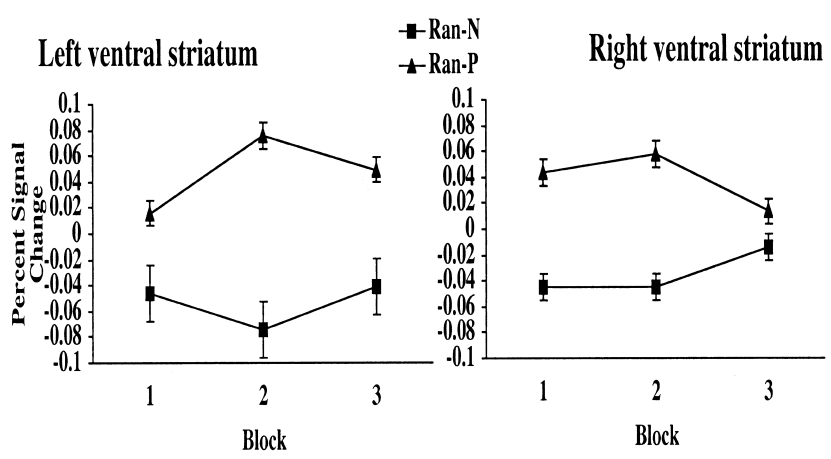

Figure 7. The percentage of signal change in the right and left head of the caudate and right and left ventral striatum across blocks for random stimuli receiving positive feedback and random stimuli receiving negative feedback (Ran-P and Ran-N) is shown.

greater in the right and left head of the caudate and right and left ventral striatum than in the other areas of the striatum. Paired $t$ tests with the difference (percentage of signal change during Ran$\mathrm{P}$ - percentage of signal change during Ran-N) as dependent measure showed that the difference in the left head of the caudate was significantly greater than the difference in the right head of the caudate $\left[t_{(14)}=2.42 ; p<0.05\right]$, right body and tail of the caudate $\left[t_{(14)}=2.87 ; p<0.05\right]$, left body and tail of the caudate $\left[t_{(14)}=2.70 ; p<0.05\right]$, and right putamen $\left[t_{(14)}=2.22 ; p<\right.$ $0.05]$. The difference in the right head of the caudate was significantly greater than that in the right body and tail $\left[t\left(_{14}\right)=2.15\right.$; $p<0.05]$. The difference in the left ventral striatum was significantly greater than the difference in the right body and tail $\left[t_{(14)}=2.7 ; p<0.05\right]$ and left body and tail of the caudate $\left[t_{(14)}=\right.$ $2.5 ; p<0.05]$. As shown in Figure 7, the difference between the head of the caudate and right ventral caudate activity in Ran-P and Ran- $\mathrm{N}$ trials decreased with learning; the difference was greater in blocks 1 and 2 than in block 3 .

\section{Whole-brain analysis of cortex}

Areas associated with classification: Det-CP and Prob-CP $>$ Base The goal of this contrast was to identify cortical areas active during classification of deterministic and probabilistic stimuli in comparison with the baseline task. A conjunction analysis was performed, which identified areas that were active for both stimulus types in comparison with baseline. As shown in Table 5, Det-CP and Prob-CP both activated the left insula, posterior cingulate, and precuneus. Several areas were more active during baseline trials than during classification, including bilateral medial frontal areas, the bilateral superior temporal gyri, the right inferior parietal lobe, and the left cerebellum. 
Table 5. Areas of activation during correct classification versus baseline outside of the striatum

\begin{tabular}{|c|c|c|c|c|c|c|}
\hline & $\mathrm{BA}$ & $x$ & $y$ & $z$ & Voxels & $t \max$ \\
\hline \multicolumn{7}{|l|}{ Classification $>$ baseline } \\
\hline Right insula & & 32 & 6 & -6 & 1237 & 4.5 \\
\hline Left insula & & -27 & 18 & 6 & 1684 & 4.5 \\
\hline Left posterior cingulate & 31 & -21 & -37 & 38 & 1956 & 5.8 \\
\hline Bilateral posterior cingulate/precuneus & 31 & -4 & -37 & 31 & 1174 & 4.4 \\
\hline \multicolumn{7}{|l|}{ Baseline $>$ classification } \\
\hline Right inferior parietal lobule & 40 & 49 & -33 & 36 & 8431 & 5.4 \\
\hline $\begin{array}{l}\text { Right superior temporal and inferior } \\
\text { frontal gyri }\end{array}$ & $43,44,6,22$ & 57 & 3 & 20 & 4867 & 5.3 \\
\hline $\begin{array}{l}\text { Left superior temporal and inferior } \\
\text { frontal gyri }\end{array}$ & $43,40,6,22$ & -59 & 4 & 14 & 2566 & 5.2 \\
\hline Bilateral medial frontal & 9,32 & -7 & 32 & 32 & 3334 & 4.7 \\
\hline Cerebellum & & 6 & -50 & -15 & 307 & 5.5 \\
\hline
\end{tabular}

Clusters are based on a random effects conjunction analysis of two contrasts: deterministic correct (1) versus baseline ( -1 ) and probabilistic correct (1) versu baseline (-1). $x, y, z$, Talairach coordinates (Talairach and Tournoux, 1988) of the central voxel of the activated cluster. $t$ max, $t$ value of the maximally activated voxel within the cluster.

Table 6. Areas of activation affected by stimulus probability and learnability outside of the striatum

\begin{tabular}{|c|c|c|c|c|c|c|}
\hline & $\mathrm{BA}$ & $x$ & $y$ & $z$ & Voxels & $t \max$ \\
\hline \multicolumn{7}{|l|}{ Det- $C P>$ Prob-CP } \\
\hline Left superior frontal gyrus/frontal pole & 8 & -11 & 44 & 45 & 357 & 4.5 \\
\hline \multicolumn{7}{|l|}{ Prob-CP $>$ Det- $C P$} \\
\hline Left middle temporal gyrus & 39 & -37 & -64 & 24 & 375 & 3.8 \\
\hline Left lingual gyrus & 19 & -6 & -56 & 0 & 828 & 4.3 \\
\hline \multicolumn{7}{|l|}{ Prob-CP $>$ Ran-P } \\
\hline \multicolumn{7}{|l|}{ No significant clusters } \\
\hline \multicolumn{7}{|l|}{ Det-CP $>$ Ran-P } \\
\hline \multicolumn{7}{|l|}{ No significant clusters } \\
\hline \multicolumn{7}{|l|}{ Ran-P $>$ Prob-CP } \\
\hline Left superior frontal gyrus/frontal pole & 8,9 & -9 & 40 & 36 & 4150 & 5.0 \\
\hline Left supramarginal and angular gyri & 39,40 & -34 & -55 & 35 & 2357 & 5.0 \\
\hline Right supramarginal and angular gyri & 39,40 & 40 & -53 & 34 & 1685 & 4.8 \\
\hline Right precuneus and superior occipital gyrus & 19 & 37 & -76 & 41 & 1958 & 5.2 \\
\hline \multicolumn{7}{|l|}{ Ran-P $>$ Det-CP } \\
\hline Left supramarginal and angular gyri & 39,40 & -32 & -56 & 36 & 2223 & 4.8 \\
\hline Left superior occipital gyri & 19 & -36 & -83 & 26 & 1608 & 4.7 \\
\hline Right supramarginal, angular and sup. occ. Gyri & $19,39,40$ & 36 & -69 & 38 & 3054 & 5.3 \\
\hline Bilateral cerebellum and lingual gyri & 18 & 0 & -57 & -2 & 2855 & 4.7 \\
\hline Left middle temporal gyrus & 39 & -35 & -44 & 9 & 946 & 5.0 \\
\hline
\end{tabular}

$x, y, z$, Talairach coordinates (Talairach and Tournoux, 1988) of the central voxel of the activated cluster. $t$ max, $t$ value of the maximally activated voxel within the cluster.

Effect of stimulus contingency: pairwise comparisons between Det-CP, Prob-CP, and Ran-P

Differences in neural activity in processing deterministic relationships in contrast to probabilistic relationships were examined by comparing Det-CP trials with Prob-CP trials. As listed in Table 6 and shown in Figure 8a, deterministic stimuli led to greater activity than probabilistic in a small area of the left frontal pole. Conversely, probabilistic stimuli led to greater activity in the left lingual gyrus and left middle temporal gyrus.

Conditions in which successful learning occurred (Det-CP and Prob-CP) were compared with Ran-P, in which no classification learning could occur, to examine the effects of the presence or absence of learned relationships on brain activity during classification. No areas outside of the striatum were significantly activated in the Det-CP $>$ Ran-P or Prob-CP $>$ Ran-P comparisons. Conversely, several brain areas were more active in the random condition. In both Ran-P $>$ Prob-CP and Ran-P $>$ Det$\mathrm{CP}$, there were significantly activated clusters in the bilateral superior occipital, supramarginal, and angular gyri. A large area of the left frontal pole, including both lateral and medial aspects of the superior frontal gyrus, was active only in the Ran-P >
Prob-CP comparison. Bilateral cerebellum and lingual gyri areas and the left lateral middle temporal gyrus were active only in the Ran-P > Det-CP comparison.

Because frontal activation has been found in other categorization tasks, the pattern of activity in the left frontal pole was examined in more detail using an ROI analysis. The ROI was defined based on the contrast between Det-CP and Prob-CP with the threshold set to $p<0.0064$. The resulting cluster was 357 voxels, centered at $x=-11, y=44$, and $z=45$, and was a subsection of the cluster activated in the Ran-P > Prob-CP comparison. As shown in Figure 9, the greatest differences between the conditions occurred in block 2, with Det-CP more active than Ran-P, which in turn was more active than ProbCP. Activity in the left frontal pole was significantly correlated with overall accuracy when probabilistic stimuli were classified $(r=0.60 ; p<0.05)$ but not when deterministic $(r=0.30 ; p>0.1)$ or random $(r=0.02 ; p>0.1)$ stimuli were classified.

Effect of feedback: Ran-P versus Ran-N As shown in Table 7, several areas were more active in Ran-P than Ran- $\mathrm{N}$, particularly a large area of the right hippocampus (Fig. 8b) and smaller areas of the left parahippocampal gyrus and left fusiform gyrus. In addition, there were activations in the right anterior cingulate and the bilateral precuneus.

Because previous studies have shown a negative relationship between caudate and hippocampal activity, we defined a functionally based ROI of the right hippocampal cluster using a threshold of $p<0.001$. The resulting ROI was 1561 voxels, centered at $x=24, y=-20$, and $z=-4$. The activity in the right hippocampus during classification (Det-CP and Prob-CP) trials did not correlate significantly with activity in the right body $(r=-0.12)$, left body $(r=-0.03)$, right putamen $(r=0.04)$, left putamen $(r=0.00)$, right head $(r=0.06)$, left head $(r=-0.01)$, right ventral striatum $(r=0.12)$, and left ventral striatum $(r=0.23)$ ( $p>0.1$ for all). As shown in Table 4, activity in the right hippocampus activity correlated negatively with accuracy during the first half of the experiment, indicating that people who recruited this area were slower at learning in the early stages. As shown in Figure 10, activity in the right hippocampus for Ran-P trials was greater than that for Ran-N trials in blocks 1 and 2 but not block 3 .

\section{Discussion}

Successful learning and the body and tail of the caudate and putamen

In this study, we found a close association between learning to classify stimuli and activity in the bilateral body and tail of the caudate nucleus. First, the body and tail of the caudate was consistently active on trials in which participants correctly classified stimuli regardless of positive or negative feedback, including the 


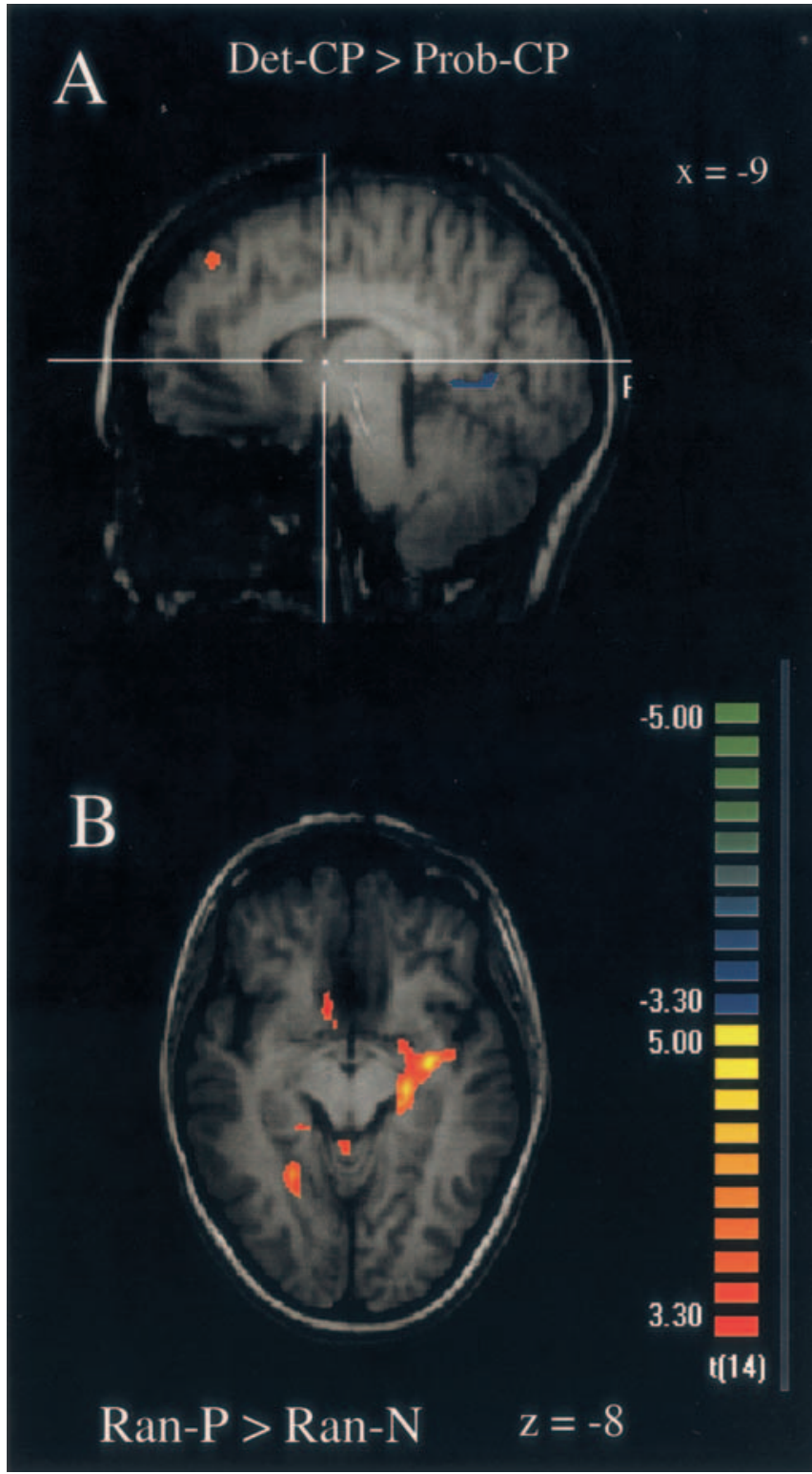

Figure 8. A, Areas of the left frontal pole that were more active in Det-CP than Prob-CP (positive $t$ values; red-yellow scale) and areas of the left lingual gyrus that were more active in Prob-CP than Det-CP (negative $t$ values; blue-green scale). $\boldsymbol{B}$, Right hippocampal and left parahippocampal gyrus clusters that were more active in Ran-P than Ran-N (positive $t$ values; redyellow scale).

$\mathrm{ROI}$ analysis comparing Prob-CP and Det-CP with baseline and Prob-CP with Ran-P. Second, activity increased across trials as learning occurred. Third, activity correlated significantly with accuracy during the second half, indicating that participants who recruited the body and tail of the caudate learned better than those who did not. The involvement of the body and tail of the caudate in learning is consistent with a large body of research with monkeys (Brown et al., 1995; Levy et al., 1997; Teng et al., 2000; Fernandez-Ruiz et al., 2001). Performance on complex visual stimulus-outcome tasks is impaired in patients with compromised striatal functioning (Knowlton et al., 1996a,b; Filoteo et al., 2001, 2005a; Keri et al., 2001, 2002; Maddox and Filoteo, 2001; Witt et al., 2002; Myers et al., 2003; Sage et al., 2003; Shohamy et al., 2004). It is also consistent with the theory of Ashby et

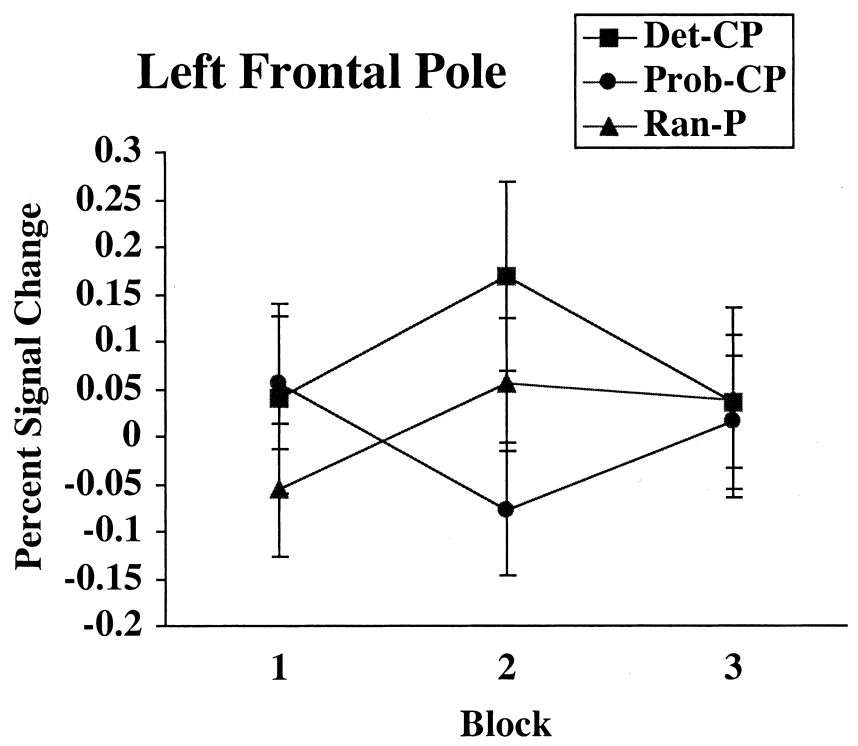

Figure 9. The percentage of signal change in the left frontal pole across blocks for deterministic (Det-CP), probabilistic (Prob-CP), and random (Ran-P) stimuli receiving positive feedback is shown.

al. (1998) and Ashby and Casale (2003) that the "visual" corticostriatal loop underlies human classification learning via feedback.

ROI analyses indicated that the putamen was significantly activated during classification learning, with a pattern of activity more similar to the pattern in the body and tail of the caudate than the head of the caudate. Putamen activity was greater in classification trials than baseline trials, increased with learning, and correlated positively with classification accuracy. Previous studies have reported putamen activity associated with classification learning (Table 1).

Executive functions and the head of the caudate

Previous functional imaging research on human categorization has reported activation in the head of the caudate rather than the body and tail regions. In contrast, we found that activity in the head of the caudate was not related to learning to categorize but instead was related to feedback processing. The right and left heads were more active when receiving positive feedback than negative feedback during classification of random stimuli. Although other areas of the dorsal striatum were also significantly more active during Ran- $\mathrm{P}$ than Ran- $\mathrm{N}$, the modulation was strongest in the head. Sensitivity of the head of the caudate to feedback is consistent with its interaction with the dorsolateral prefrontal cortex in the cognitive loop.

\section{Effects of stimulus outcome contingencies}

Probabilistic stimuli led to greater activation of the bilateral body and tail of the caudate and extrastriate visual areas than deterministic stimuli. Conversely, deterministic stimuli led to greater activity in the left superior frontal gyrus. Many of the previous stimulus-outcome learning studies that have shown caudate activation have indicated probabilistic relationships between stimulus and outcome because of category overlap or stochastic design (Table 1). In contrast, Bischoff-Grethe et al. (2001) found greater recruitment of the head of the caudate for learning deterministic than probabilistic sequences.

Activation differed as a result of stimulus-outcome contingencies in two visual processing areas: the lingual gyrus and left 
Table 7. Areas of activation affected by feedback outside of the striatum: random trials with positive feedback versus negative feedback

\begin{tabular}{|c|c|c|c|c|c|c|}
\hline & $\mathrm{BA}$ & $x$ & $y$ & $z$ & Voxels & $t \max$ \\
\hline \multicolumn{7}{|l|}{ Ran-P $>$ Ran-N } \\
\hline Right hippocampus & & 24 & -20 & -4 & 4330 & 6.7 \\
\hline Left parahippocampal gyrus & 27,30 & -12 & -35 & 2 & 3035 & 5.0 \\
\hline Left fusiform gyrus & 36 & -16 & -30 & -13 & 735 & 4.7 \\
\hline Left fusiform gyrus & 19 & -24 & -50 & -10 & 689 & 5.4 \\
\hline Left superior frontal & 6 & -16 & 14 & 54 & 3884 & 4.6 \\
\hline Right anterior cingulate & 32 & 15 & 24 & 41 & 7250 & 5.0 \\
\hline Left posterior cingulate & 7 & -15 & -48 & 41 & 5553 & 5.7 \\
\hline Right posterior cingulate & 7 & 19 & -41 & 46 & 1513 & 5.5 \\
\hline Bilateral precuneus & 7 & -4 & -76 & 43 & 11179 & 6.6 \\
\hline
\end{tabular}

Ran-N > Ran-P, No significant clusters.

\section{Right Hippocampus}

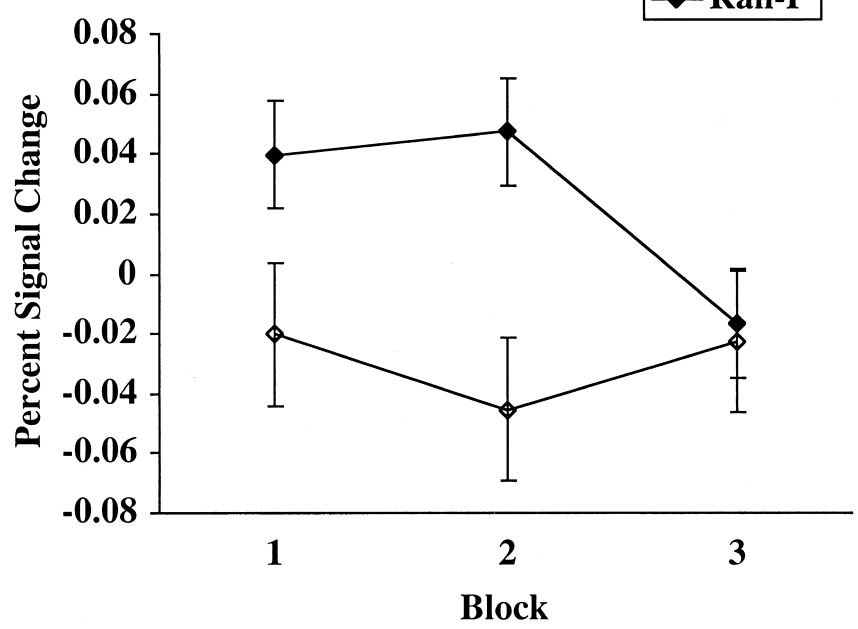

Figure 10. The percentage of signal change in the right hippocampus across blocks for random stimuli receiving positive feedback (Ran-P) and random stimuli receiving negative feedback (Ran-N) is shown.

middle temporal gyrus. Activity in both areas was greater for probabilistic and random stimuli than deterministic stimuli. The visual corticostriatal loop connects the body and tail of the caudate with inferior temporal lobe visual processing areas. Research in monkeys has shown that visual discrimination learning relies on the interaction of the body and tail of the caudate with temporal lobe visual processing area TE (Gaffan and Eacott, 1995; Buffalo et al., 1998, 1999).

The left superior frontal gyrus was more active during classification of random and deterministic than probabilistic stimuli. This area is approximately equivalent to the presupplementary motor area and encompasses portions of Brodmann's area (BA) 8 and BA 9. The differences between conditions were most apparent on block 2, with deterministic stimuli showing the most activity, random stimuli showing intermediate amounts of activity, and probabilistic stimuli showing the least activity. Activity in the left superior frontal gyrus during probabilistic trials correlated positively with accuracy. Therefore, overall, participants tended to have lower levels of activity on probabilistic trials, but participants with relatively high activity in this area were more successful at learning. Volz et al. (2003) found that activity in a similar location was associated with making predictions under uncertainty, with higher activity as uncertainty increased. In contrast, in our study, activity was lowest for the probabilistic stimuli.

\section{The medial temporal lobe and its} interactions with the striatum

Activity in the medial temporal lobe was not associated with successful categorization learning in this study. The only comparison in which hippocampal activity was found was in Ran-P $>$ Ran-N, indicating that the hippocampus was more active when participants were told they had correctly classified a random stimulus than when told they were incorrect. However, previous research has shown an antagonistic relationship between striatal and medial temporal lobe learning systems, which led us to examine whether such antagonism was apparent in our data. Poldrack et al. (2001) compared learning in a probabilistic classification task with feedback and via explicit instruction. The caudate was active in the task with feedback, whereas the medial temporal lobe was active in the instructed version. Furthermore, caudate and medial temporal activity was negatively correlated. In contrast, we found that hippocampal and caudate activity did not correlate, either positively or negatively. Some antagonism was indirectly observed in correlation analyses between brain activation and learning. Caudate activity was positively correlated with late classification accuracy, whereas hippocampal activity was negatively correlated with early classification accuracy. Thus, participants who successfully learned tended to activate the body and tail of the caudate and not activate the hippocampus to a greater degree than participants who were less successful.

\section{Task complexity and structure}

Studies that indicate caudate involvement in stimulus-outcome learning have several features in common, including learning with feedback and mapping stimuli to distinct motor responses. However, the tasks vary greatly in complexity, as shown in Table 1. In some tasks, participants learn to respond to four different stimuli with four different finger presses. In other tasks, stimuli are generated according to complicated patterns or rules, and novel stimuli are presented on each trial. The present study is intermediate in complexity, using eight different stimuli and two different categories. It should be noted that the stimuli were not related to other stimuli within the category, and thus participants were not able to abstract a rule, pattern, or prototype or make similarity-based judgments (Seger, 1994; Ashby and Casale, 2003).

\section{Feedback processing in dorsal and ventral striatum}

In the present study, when classifying random stimuli, positive feedback led to greater activity than negative feedback across the basal ganglia, particularly in the right and left heads of the caudate and right and left ventral striatum. Delgado et al. (2004) found the same pattern of activation in a gambling task with random stimulus-reward contingencies. A pattern that emerges across studies is that the dorsal head of the caudate is sensitive to feedback or reward only when it is the result of an action (Tremblay et al., 1998; Lauwereyns et al., 2002; O’Doherty et al., 2004; Tricomi et al., 2004).

We found a similar pattern of activity in the ventral striatum, which is consistent with a wide variety of studies showing reward sensitivity in this area (Ravel et al., 2001; McClure et al., 2003), regardless of whether the feedback was linked to an action (O'Doherty et al., 2004). The ventral striatum receives input from 
dopaminergic cells of the midbrain, particularly in the ventral tegmental area, that are also sensitive to reward (Fiorillo et al., 2003). Aron et al. (2004) found more activity for negative feedback than positive feedback in the midbrain during a probabilistic classification task.

\section{Role of caudate in feedback-related learning}

We found that all areas of the striatum were sensitive to feedback, particularly when expectations about feedback were violated. The sensitivity of areas of caudate that were important for classification (e.g., the body and tail of the caudate) to feedback is consistent with theories that the striatum may play a general role in reward-mediated learning (Ashby and Casale, 2003). Behavioral and neuropsychological studies show that categorization learning is dependent on the presence of feedback. Learning is worse in observational conditions than feedback conditions (Ashby et al., 2002) and is impaired when feedback is delayed (Maddox et al., 2003). Classification tasks impaired in Huntington's or Parkinson's diseases typically involve feedback; patients with Parkinson's disease learn normally on observational tasks (Reber and Squire, 1999; Shohamy et al., 2004).

In conclusion, we have shown two ways that the caudate nucleus contributes to classification learning. The bilateral body and tail is associated with successful classification learning, and the head of the caudate is associated with processing feedback. These results underline the importance of accounting for different levels of feedback in learning tasks to more accurately separate brain areas involved in memory representations from areas involved in executive functions.

\section{References}

Aizenstein HJ, MacDonald AW, Stenger VA, Nebes RD, Larson JK, Ursu S, Carter CS (2000) Complementary category learning systems identified using event-related functional MRI. J Cogn Neurosci 12:977-987.

Aron AR, Shohamy D, Clark J, Myers C, Gluck MA, Poldrack RA (2004) Human midbrain sensitivity to cognitive feedback and uncertainty during classification learning. J Neurophysiol 92:1144-1152.

Ashby FG, Casale MB (2003) The cognitive neuroscience of implicit category learning. In: Attention and implicit learning (Jimenez L, ed), pp 109-141. Amsterdam: John Benjamins Publishing.

Ashby FG, Alfonso-Reese LA, Turken AU, Waldron EM (1998) A neuropsychological theory of multiple systems in category learning. Psychol Rev 105:442-481.

Ashby FG, Maddox WT, Bohil CJ (2002) Observational versus feedback training in rule-based and information-integration category learning. Mem Cogn 30:666-677.

Berns GS, McClure SM, Pagnoni G, Montague PR (2001) Predictability modulates human brain response to reward. J Neurosci 21:2793-2798.

Bischoff-Grethe A, Martin M, Moa H, Berns GS (2001) The context of uncertainty modulates the subcortical response to predictability. J Cogn Neurosci 13:986-993.

Brown VJ, Desimone R, Mishkin M (1995) Responses of cell in the tail of the caudate nucleus during visual discrimination learning. J Neurophysiol 74:1083-1094.

Buffalo EA, Stefanacci L, Squire LR, Zola SM (1998) A reexamination of the concurrent discrimination learning task: the importance of anterior inferotemporal cortex area TE. Behav Neurosci 112:3-14.

Buffalo EA, Ramus SJ, Clark RE, Teng E, Squire LR, Zola SM (1999) Dissociation between the effects of damage to perirhinal cortex and area TE. Learn Mem 6:572-599.

Cools R, Clark L, Owen AM, Robbins TW (2002) Defining the neural mechanisms of probabilistic reversal learning using event-related functional magnetic resonance imaging. J Neurosci 22:4563-4567.

Cools R, Clark L, Robbins TW (2004) Differential responses in human striatum and prefrontal cortex to changes in object and rule relevance. J Neurosci 24:1129-1135.

Dale AM (1999) Optimal experimental design for event-related fMRI. Hum Brain Mapp 8:109-114.
Delgado MR, Stenger VA, Fiez JA (2004) Motivation-dependent responses in the human caudate nucleus. Cereb Cortex 14:1022-1030.

Fernandez-Ruiz J, Wang J, Aigner TG, Mishkin M (2001) Visual habit formation in monkeys with neurotoxic lesions of the ventrocaudal neostriatum. Proc Natl Acad Sci USA 98:4196-4201.

Filoteo JV, Maddox WT, Davis J (2001) A possible role of the striatum in linear and nonlinear categorization rule learning: evidence from patients with Huntington's disease. Behav Neurosci 115:786-798.

Filoteo JV, Maddox WT, Simmons AN, Ing AD, Cagigas XE, Matthews S, Paulus MP (2005a) Cortical and subcortical brain regions involved in rule-based category learning. NeuroReport 16:111-115.

Filoteo JV, Maddox WT, Salmon DP, Song DD (2005b) Informationintegration category learning in patients with striatal dysfunction. Neuropsychology, in press.

Fiorillo CD, Tobler PN, Schulz W (2003) Discrete coding of reward probability and uncertainty by dopamine neurons. Science 299:1898-1902.

Gaffan D, Eacott MJ (1995) Visual learning for an auditory secondary reinforcer by macaques is intact after uncinate fascicle section: indirect evidence for the involvement of the corpus striatum. Eur J Neurosci 7:1866-1871.

Glover GH, Law CS (2001) Spiral-in/out BOLD fMRI for increased SNR and reduced suspectibility artifacts. Magn Reson Med 46:515-522.

Haber SN, Fudge JL, McFarland NR (2000) Striatonigrostriatal pathways in primates form an ascending spiral from the shell to the dorsolateral striatum. J Neurosci 20:2369-2382.

Haruno M, Kuroda T, Doya K, Toyama K, Kimura M, Samejima K, Imamizu H, Kawato M (2004) A neural correlate of reward-based behavioral learning in caudate nucleus: a functional magnetic resonance imaging study of a stochastic decision task. J Neurosci 24:1660-1665.

Hopkins RO, Myers CE, Shohamy D, Grossman S, Gluck M (2004) Impaired probabilistic category learning in hypoxic subjects with hippocampal damage. Neuropsychologia 42:524-535.

Keri S, Szlobodnyik C, Benedek G, Janka Z, Gadoros J (2001) Probabilistic classification learning in Tourette syndrome. Neuropsychologia 1351:1-7.

Keri S, Beniczky S, Voros E, Janka Z, Benedek G, Vecsel L (2002) Dissociation between attentional set shifting and habit learning: a longitudinal case study. Neurocase 8:219-225.

Knowlton BK, Squire LR, Gluck MA (1994) Probabilistic classification learning in amnesia. Learn Mem 1:106-120.

Knowlton BK, Mangels JA, Squire LR (1996a) A neostriatal habit learning system in humans. Science 273:1399-1402.

Knowlton BK, Squire LR, Paulsen JS, Swerdlow NR, Swenson M, Butters N (1996b) Dissociations within nondeclarative memory in Huntington's disease. Neuropsychology 10:538-548.

Lauwereyns J, Watanabe K, Coe B, Hikosaka O (2002) A neuronal correlate of response bias in monkey caudate nucleus. Nature 418:413-417.

Lawrence AD, Sahakian BJ, Robbins TW (1998) Cognitive functions and corticostriatal circuits: insights from Huntington's disease. Trends Cogn Sci 2:379-388.

Levy R, Friedman HR, Davachi L, Goldman-Rakic PS (1997) Differential activation of the caudate nucleus in primates performing spatial and nonspatial working memory tasks. J Neurosci 17:3870-3882.

Lieberman MD, Chang GY, Chiao J, Bookheimer SY, Knowlton BJ (2004) An event-related fMRI study of artificial grammar learning in a balanced chunk strength design. J Cogn Neurosci 16:427-438.

Maddox WT, Filoteo JV (2001) Striatal contributions to category learning: quantitative modeling of simple linear and complex non-linear rule learning in patients with Parkinson's disease. J Int Neuropsychol Soc 7:710-727.

Maddox WT, Ashby FG, Bohil CJ (2003) Delayed feedback effects on rulebased and information-integration category learning. J Exp Psychol Learn Mem Cogn 29:650-662.

McClure SM, Berns GS, Montague PR (2003) Temporal prediction errors in a passive learning task activate human striatum. Neuron 38:339-346.

McClure SM, Laibson DI, Loewenstein G, Cohen JD (2004) Separate neural systems value immediate and delayed monetary rewards. Science 306:503-507.

Middleton FA, Strick PL (1996) The temporal lobe is a target of output from the basal ganglia. Proc Natl Acad Sci USA 93:8683-8687.

Mink JW (1996) The basal ganglia: focused selection and inhibition of competing motor programs. Prog Neurobiol 50:381-425. 
Monchi O, Petrides M, Petre V, Worsley K, Dagher A (2001) Wisconsin card sorting revisited: distinct neural circuits participating in different stages of the task identified by event-related functional magnetic resonance imaging. J Neurosci 21:7733-7741.

Monchi O, Petrides M, Doyon J, Postuma RB, Worsley K, Dagher A (2004) Neural bases of set-shifting deficits in Parkinson's disease. J Neurosci 24:702-710.

Myers CE, Shohomy D, Gluck MA, Grossman S, Kluger A, Ferris S, Golomb J, Schnirman G, Schwartz R (2003) Dissociating hippocampal versus basal ganglia contributions to learning and transfer. J Cogn Neurosci 15:185-193.

O’Doherty JP, Dayan P, Friston K, Critchley H, Dolan RJ (2003) Temporal difference models and reward-related learning in the human brain. Neuron 28:329-337.

O’Doherty JP, Dayan P, Schulz J, Deichmann R, Friston K, Dolan RJ (2004) Dissociable roles of ventral and dorsal striatum in instrumental conditioning. Science 304:452-454.

Packard MG, Knowlton BJ (2002) Learning and memory functions of the basal ganglia. Annu Rev Neurosci 25:563-593.

Poldrack RA, Packard MG (2003) Competition among multiple memory systems: converging evidence from animal and human brain studies. Neuropsychologia 41:245-251.

Poldrack RA, Prabhakaran V, Seger CA, Gabrieli JDE (1999) Striatal activation during cognitive skill learning. Neuropsychology 13:564-574.

Poldrack RA, Clark J, Pare-Blagoev EJ, Shohamy D, Creso Moyano J, Myers C, Gluck MA (2001) Interactive memory systems in the human brain. Nature 414:546-550.

Preston AR, Thomason ME, Ochsner KN, Cooper JC, Glover GH (2004) Comparison of spiral-in/out and spiral-out BOLD fMRI at 1.5T and 3T. NeuroImage 21:291-301.

Ravel S, Sardo P, Legallet E, Apicella P (2001) Reward unpredictability inside and outside of a task context as a determinant of the responses of tonically active neurons in the monkey striatum. J Neurosci 21:5370-5739.

Reber PJ, Squire LR (1999) Intact learning of artificial grammars and intact category learning by patients with Parkinson's disease. Behav Neurosci 113:235-242.

Reber PJ, Knowlton BJ, Squire LR (1996) Dissociable properties of memory: differences in the flexibility of declarative and nondeclarative knowledge. Behav Neurosci 110:861-871.

Reber PJ, Stark CEL, Squire LR (1998a) Cortical areas supporting category learning identified using functional MRI. Proc Natl Acad Sci USA 95:747-750.

Reber PJ, Stark CEL, Squire LR (1998b) Contrasting cortical activity associated with category memory and recognition memory. Learn Mem 5:420-428.

Reber PJ, Gitelman DR, Parrish TB, Mesulam M-M (2003) Dissociating conscious and nonconscious knowledge with fMRI. J Cogn Neurosci 15:574-685.

Rogers RD, Andrews TC, Grasby PM, Brooks DJ, Robbins TW (2000) Con- trasting cortical and subcortical activations produced by attentional-set shifting and reversal learning in humans. J Cogn Neurosci 12:142-162.

Sage JR, Anagnostaras SG, Mitchell S, Bronstein JM, De Salles A, Masterman D, Knowlton BJ (2003) Analysis of probabilistic classification learning in patients with Parkinson's disease before and after pallidotomy surgery. Learn Mem 10:226-236.

Seger CA (1994) Implicit learning. Psychol Bull 115:163-196.

Seger CA, Cincotta CM (2002) Striatal activation in concept learning. Cogn Affect Behav Neurosci 2:149-161.

Seger CA, Poldrack RA, Prabhakaran V, Zhao M, Glover G, Gabrieli JDE (2000) Hemispheric asymmetries and individual differences in visual concept learning as measured by functional MRI. Neuropsychologia 38:1316-1324.

Shohamy D, Myers CE, Grossman S, Sage J, Gluck MA, Poldrack RA (2004) Cortico-striatal contributions to feedback-based learning: converging data from neuroimaging and neuropsychology. Brain 127:851-859.

Smith EE, Patalano AL, Jonides J (1998) Alternative strategies of categorization. Cognition 65:167-196.

Talairach J, Tournoux P (1988) Co-planar stereotactic atlas of the human brain. New York: Thieme.

Teng E, Stefanacci L, Squire LR, Zola SM (2000) Contrasting effects on discrimination learning after hippocampal lesions and conjoint hippocampal-caudate lesions in monkeys. J Neurosci 20:3853-3863.

Toni I, Passingham RE (1999) Prefrontal-basal ganglia pathways are involved in the learning of arbitrary visuomotor associations: a PET study. Exp Brain Res 127:19-32.

Toni I, Ramnani N, Josephs O, Ashburner J, Passingham RE (2001a) Learning arbitrary visuo-motor associations: temporal dynamic of brain activity. NeuroImage 14:1048-1057.

Toni I, Rushworth MFS, Passingham RE (2001b) Neural correlates of visuomotor associations: spatial rules compared with arbitrary rules. Exp Brain Res 141:359-369.

Toni I, Rowe J, Stephan KE, Passingham RE (2002) Changes of corticostriatal effective connectivity during visuomotor learning. Cereb Cortex 12:1040-1047.

Tremblay L, Hollerman JR, Shultz W (1998) Modifications of reward expectation-related neuronal activity during learning in primate striatum. J Neurophysiol 80:964-977.

Tricomi EM, Delgado MR, Fiez JA (2004) Modulation of caudate activity by action contingency. Neuron 41:281-292.

Vogels R, Sary G, Dupont P, Orban GA (2002) Human brain regions involved in visual categorization. NeuroImage 16:401-414.

Volz KG, Schubotz RI, von Cramon DY (2003) Predicting events of varying probability: uncertainty investigated. NeuroImage 19:271-280.

Wise SP, Murray EA (2000) Arbitrary associations between antecedents and actions. Trends Neurosci 23:271-276.

Wise SP, Murray EA, Gerfew CR (1996) The frontal cortex-basal ganglia system in primates. Crit Rev Neurobiol 10:317-356.

Witt K, Nuhsman A, Deuschl G (2002) Dissociation of habit-learning in Parkinson's and cerebellar disease. J Cogn Neurosci 14:493-499. 